\title{
Preface
}

\section{Cardiothoracic Anesthesia and Critical Care: An Ever-Changing (and Evolving) Field}

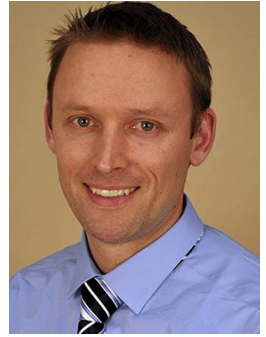

Karsten Bartels, MD, PhD

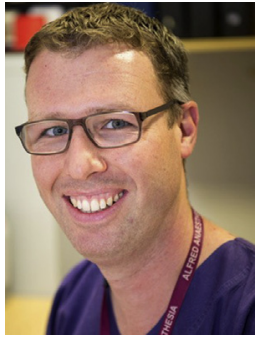

Stefan J.M. Dieleman, MD, PhD Editors

Perioperative care for patients undergoing cardiothoracic surgery has evolved significantly since the first open-heart procedures were performed in the 1950s. Nowadays, the majority of cardiac surgical procedures are considered "routine" operations that, given the presence of significant comorbid disease in most of these patients, come with a rather acceptable risk of major perioperative complications. As a result, longterm outcomes for most patients are usually very good, too.

Inevitably, having consistently good results leads to further advancements in the field. Patients who would (or could) not be considered for cardiac surgery 10 to 20 years ago because of severe comorbid disease, advanced age, or congenital defects, are now presenting to our operating rooms. Also, several new interventional techniques have emerged that present the anesthesiologist with the challenge to further evolve their practice in order to provide state-of-the-art perioperative care for these patients. However, despite the great potential of all these advancements, it also requires an increased awareness of the ethical aspects of such developments. While it may be technically possible to provide certain interventions to an increasingly sick patient population, careful consideration should be given to patient selection.

This issue of Anesthesiology Clinics focuses on several aspects of the recent developments in the area of cardiothoracic anesthesiology and critical care. Multiple experts from several fields have contributed to a variety of articles to provide an update on several exciting developments in techniques, optimization of clinical care, as well as basic science. Evolving techniques that are discussed include endovascular approaches to thoracic aortic repair, ${ }^{1}$ modulation of ventricular excitability, ${ }^{2}$ catheterbased cardiac valve procedures, ${ }^{3}$ and ex vivo optimization of donor hearts and lungs, ${ }^{4}$ 
respectively. In a separate article, the ethical dilemmas of the increasing technical possibilities in the field of mechanical circulatory support are considered. ${ }^{5}$ The articles on optimization of clinical care encompass a review of both echocardiographic ${ }^{6}$ and catheter-based intraoperative assessment of right ventricular function, ${ }^{7}$ optimization of coagulation and blood transfusion management, ${ }^{8}$ prevention of perioperative acute kidney injury, ${ }^{9}$ as well as an overview of the aspects of perioperative management of adult patients with congenital heart disease. ${ }^{10}$ Two articles focused on emerging basic science will outline the effects of cardiac surgery on mitochondrial ${ }^{11}$ and blood-brain barrier function, ${ }^{12}$ respectively. Finally, an update is provided on recent, upcoming, and highly needed clinical trials, ${ }^{13}$ in order to make cardiac anesthesiology an even more evidence-based field.

While we cannot claim that this issue of Anesthesiology Clinics provides an all-encompassing picture of developments in the field of cardiothoracic anesthesiology and critical care, we believe that the authors offer a thought-provoking summary of exciting insights driven by leaders in our field. We would like to sincerely thank the authors of this issue for providing their valuable time to share this progress with our readers, and we hope readers will enjoy their contributions as much as we did.

Karsten Bartels, MD, PhD Department of Anesthesiology University of Colorado 12401 East 17th Avenue, MS B-113 Aurora, CO 80045, USA

Stefan J.M. Dieleman, MD, PhD Department of Anaesthesia Westmead Hospital

CNR Hawkesbury Road/Darcy Road Westmead, NSW 2145, Australia

E-mail addresses: karsten.bartels@cuanschutz.edu (K. Bartels) stefandieleman@me.com (S.J.M. Dieleman)

\section{REFERENCES}

1. Cheruku S, Huang N, Meinhardt K, et al. Anesthetic management for endovascular repair of the thoracic aorta. Anesthesiol Clin 2019;37(4):593-607.

2. Howard-Quijano K, Kuwabara Y. Modulating perioperative ventricular excitability. Anesthesiol Clin 2019;37(4):609-19.

3. Ackermann MA, Ender JK. Recent developments in catheter-based cardiac procedures. Anesthesiol Clin 2019;37(4):621-38.

4. Braithwaite SA, van der Kaaij NP. New techniques for optimization of donor lungs/ hearts. Anesthesiol Clin 2019;37(4):639-60.

5. Abrams D, Curtis JR, Prager KM, et al. Ethical considerations for mechanical support. Anesthesiol Clin 2019;37(4):661-73.

6. Vandenheuvel M, Bouchez S, Wouters P, et al. Assessing right ventricular function in the perioperative setting, Part I: echo-based measurements. Anesthesiol Clin 2019;37(4):675-95.

7. Vandenheuvel M, Bouchez S, Wouters $\mathrm{P}$, et al. Assessing right ventricular function in the perioperative setting, part II-what about catheters? Anesthesiol Clin 2019;37(4):697-712. 
8. Meesters MI, von Heymann C. Optimizing perioperative blood and coagulation management during cardiac surgery. Anesthesiol Clin 2019;37(4):713-28.

9. Jacob KA, Leaf DE. Prevention of cardiac surgery-associated acute kidney injury: a review of current strategies. Anesthesiol Clin 2019;37(4):729-49.

10. Smit-Fun VM, Buhre WF. Heart failure in adult patients with congenital heart disease. Anesthesiol Clin 2019;37(4):751-68.

11. Cherry AD. Mitochondrial dysfunction in cardiac surgery. Anesthesiol Clin 2019; 37(4):769-85.

12. Hendy A, Hall R. Cardiac surgery and the blood-brain barrier. Anesthesiol Clin 2019;37(4):787-800.

13. Spence J, Mazer CD. The future directions of research in cardiac anesthesiology. Anesthesiol Clin 2019;37(4):801-13. 